\title{
Broadband X-Ray Absorption Spectra from Time-Dependent Kohn-Sham Calculations
}

\author{
Ying Zhu, ${ }^{a}$ Bushra Alam, ${ }^{b}$ and John M. Herbert ${ }^{a, b}$ \\ ${ }^{a}$ Chemical Physics Graduate Program, The Ohio State University, Columbus, Ohio, USA \\ ${ }^{b}$ Department of Chemistry and Biochemistry, The Ohio State University, Columbus, Ohio, USA
}

(Dated: June 11, 2021)

\begin{abstract}
We present a protocol for calculation of K-edge x-ray absorption spectra using time-dependent KohnSham (TDKS) calculations, also known as "real-time" time-dependent density functional theory (TDDFT). In principle, the entire absorption spectrum (at all wavelengths) can be computed via Fourier transform of the time-dependent dipole moment function, following a perturbation of the ground-state density and propagation of time-dependent Kohn-Sham molecular orbitals. In practice, very short time steps are required to obtain an accurate spectrum, which increases the cost, but the use of Padé approximants significantly reduces the length of time propagation that is required. Spectra that are well converged with respect to the corresponding linear-response (LR-)TDDFT result can be obtained with $<10$ fs of propagation time. Use of complex absorbing potentials helps to remove artifacts at high energies that otherwise result from the use of a finite atom-centered Gaussian basis set. Benchmark results, comparing TDKS to LR-TDDFT, are presented for several small molecules at the carbon and oxygen K-edges, demonstrating good agreement with experiment without the need for specialized basis sets. Whereas LR-TDDFT is a reasonable approach to obtain the near-edge structure, that approach requires hundreds of states and quickly becomes cost prohibitive for large systems, even when the core/valence separation approximation is used to remove most of the occupied states from the excitation manifold. We demonstrate the cost-effective TDKS approach by application to a copper dithiolene complex, where binding of a ligand is detectable via shifts in the sulfur K-edge.
\end{abstract}

\section{Introduction}

Quantum chemistry is currently witnessing a resurgence of interest in x-ray spectroscopy, ${ }^{1-7}$ catalyzed by the emergence of new technologies including coherent ultrahigh harmonic generation, ${ }^{8}$ providing capabilities for ultrafast time resolution at x-ray wavelengths, ${ }^{9-11}$ even with tabletop laser systems. ${ }^{12}$ This technology has enabled x-ray absorption spectroscopy (XAS) and x-ray photoelectron spectroscopy (XPS) studies of solution-phase systems, ${ }^{13-16}$ as well as surface-sensitive ultrafast spectroscopy at extreme ultraviolet (XUV) wavelengths. ${ }^{17}$ Insofar as XAS is a widely-used tool to study to interrogate bonding and oxidation state, these advances offer the possibility to study element-specific charge dynamics with ultrafast time resolution, and electronic structure theory will undoubtedly play a major role in interpreting the results. In the present work, we introduce an implementation of time-dependent KohnSham (TDKS) theory, ${ }^{18-22}$ nowadays more commonly known as "real-time" time-dependent density functional theory (RT-TDDFT), ${ }^{23,24}$ and apply it to K-edge XAS, meaning $1 \mathrm{~s} \rightarrow$ virtual excitations.

In the x-ray regime, the more conventional DFT-based approach to excited states, namely, linear-response (LR-) TDDFT, ${ }^{25-28}$ suffers from storage and computational bottlenecks that are considerably more severe as compared to the case where only a few low-lying valence excitations are desired. The computational cost of LRTDDFT scales as $\mathcal{O}\left(n o^{2} v^{2}\right),{ }^{29}$ and its memory require- ment as $\mathcal{O}($ nov), where $o$ and $v$ are the number of occupied and virtual orbitals, respectively, and $n$ is the (prohibitively large) number of excited states required to reach the x-ray regime. XAS calculations with LRTDDFT can be rendered tractable by means of an activespace approximation that includes only the core orbitals of interest, along with the full virtual space, such that core-to-valence excitations appear as the lowest states in the spectrum. In many-body theory this approximation is known as "core/valence separation", 1,30-32 whereas in LR-TDDFT it has been called the "restricted excitation window" approach, ${ }^{6,33}$ but in either case it amounts to freezing most of the occupied orbitals. For excitations at the K-edge (i.e., those originating from 1s orbitals in the occupied space), this approximation introduces negligible errors of $\pm 0.02 \mathrm{eV}, 34$ although it is less clear what the errors might be for L- or M-edge excitations. Furthermore, hundreds of states may still be required to compute anything beyond the near-edge feature of the x-ray spectrum, as demonstrated by examples presented below. For similar reasons, LR-TDDFT calculations of semiconductors or other systems with a large density of states can be prohibitively expensive, ${ }^{35-37}$ even if the corresponding ground-state DFT calculation is feasible.

For these types of systems, TDKS represents an attractive alternative as the requisite computational resources do not depend directly on the number of excited states, because the entire broadband spectrum is computed at once, via Fourier transform of the dipole autocorrelation function. The total memory requirement is only twice that of a ground-state DFT calculation. The computa- 
tional resources $d o$ depend on the energy regime of interest, as a smaller time propagation step $(\Delta t)$ is required to reach higher excitation energies, as these correspond to higher-frequency components in Fourier space. That said, for large systems the finite- $\Delta t$ error may be more easily controllable as compared to errors engendered by truncation of the excitation space in LR-TDDFT, because the former can be reduced with smaller time steps and longer propagation time, whereas enlarging the excitation space may encounter hardware (i.e., storage) limitations. Because the TDKS and LR-TDDFT approaches are formally ${ }^{20,25-27}$ and operationally ${ }^{38,39}$ equivalent in the limit of a weak perturbation to the ground-state density, the TDKS approach can be used to reveal the true TDDFT spectrum in the absence of any truncations.

The present work represents the product of a completely rewritten TDKS module in the Q-Chem software (v. 5.4), ${ }^{7}$ bringing together various improvements to enhance the efficiency of broadband spectral calculations, specifically with an eye towards XAS calculations. The resolution of the broadband TDKS spectrum is limited by the total propagation time and that cost is one or more Fock matrix constructions per time step $\Delta t$, for which values on the order of $\Delta t=0.02-0.20$ a.u. are commonly used, ${ }^{38-44}$ which equates to $0.5-5.0$ as where 1 as $=10^{-18} \mathrm{~s}$. Time propagation up to $100 \mathrm{fs}$ has been reported in a few cases, ${ }^{23,39,44}$ but more typical values are $10-30$ fs of simulated time, ${ }^{38,40-43}$ which still equates to 10,000 or more time steps. The cost of each step is roughly equivalent to that of one self-consistent field (SCF) iteration of the ground-state calculation.

The requisite propagation time is reduced here through the application of a Padé-accelerated Fourier transform technique. This method is widely used in magnetic resonance spectroscopy to treat noisy spectra with low resolution, ${ }^{45,46}$ and Padé approximants have also previously been applied in RT-TDDFT. ${ }^{47}$ Our implementation also features the use of a complex absorbing potential (CAP) in real space, which helps to remove spurious peaks that are associated with the finite atomcentered basis set. CAPs are widely used in the study of metastable resonances, ${ }^{48-50}$ but their used in the context of RT-TDDFT has previously been limited to the study of strong-field ionization dynamics, ${ }^{51-54}$ where the CAP it is necessary to absorb the outgoing electron. In the present work, we demonstrate that even in bound-state calculations the CAP eliminates spurious oscillations and is especially important at the high energies probed in XAS.

\section{Theory}

Section 22.1 provides a brief overview of TDKS theory as well as the techniques such as Padé approximants and CAPs that are used to accelerate the calculations. Because part of this work consists in a side-by-side comparison of the TDKS and LR-TDDFT methods, the theory behind the latter is briefly reviewed in Section 2.2 .

2.1. TDKS Approach. A rigorous description of TDKS theory from first principles can be found in Refs. 19-21 and the discussion here is focused on practical considerations for computing absorption spectra. The adiabatic approximation ${ }^{20}$ (i.e., locality in time) is made throughout, so that the time dependence of the exchange-correlation functional is carried strictly by the time-evolving density $\rho(\mathbf{r}, t)$, and that ground-state functionals can be used without alteration.

2.1.1. Theory. The time-dependent density $\rho(\mathbf{r}, t)$ is expressed in terms of time-dependent Kohn-Sham molecular orbitals (MOs),

$$
\rho(\mathbf{r}, t)=\sum_{k}^{\text {occ }}\left|\psi_{k}(\mathbf{r}, t)\right|^{2} .
$$

Following a perturbation to the ground-state density, these MOs propagate in time according to the TDKS equations,

$$
\mathrm{i} \hbar \frac{d \psi_{k}}{d t}=\hat{F} \psi_{k}(\mathbf{r}, t)
$$

which are one-electron analogues of the time-dependent Schrödinger equation. Here, $\hat{F}$ is the effective oneelectron Hamiltonian or Fock operator. The TDKS equations are numerically integrated in time to obtain $\left\{\psi_{k}(\mathbf{r}, t)\right\}$ from the initial ground-state MOs, $\left\{\psi_{k}(\mathbf{r}, 0)\right\}$. The latter are eigenfunctions of $\hat{F}$. A perturbative solution of eq. 2 affords the LR-TDDFT equations. ${ }^{27}$

In practice, the MOs $\left\{\psi_{k}\right\}$ are expanded in a fixed basis of atomic orbitals (AOs), and eq. 2 is equivalent to the Liouville-von Neumann equation

$$
\mathrm{i} \hbar \frac{\partial \mathbf{P}}{\partial t}=\mathbf{F P}-\mathbf{P F},
$$

where $\mathbf{P}$ is the matrix representation of $\rho$. Integration of eq. 3 affords the time-dependent density matrix,

$$
\mathbf{P}(t+\Delta t)=\mathbf{U}(t+\Delta t, t) \mathbf{P}(t) \mathbf{U}^{\dagger}(t+\Delta t, t),
$$

where $\mathbf{U}(t+\Delta t)$ is a unitary time-propagation operator for the time step $t \rightarrow t+\Delta t$. Because both $\mathbf{P}(t)$ and $\mathbf{F}(t)$ are time-dependent quantities in eq. 3, the definition of $\mathbf{U}(t+\Delta t)$ involves time-ordering of $\mathbf{F}\left(t^{\prime}\right)$ at points $t^{\prime}$ along the integration $\left(t \leq t^{\prime} \leq t+\Delta t\right)$, or else a Magnus expansion of nested commutators. ${ }^{39}$ Various forms for $\mathbf{U}(t+\Delta t)$ have been discussed in our previous work, ${ }^{39}$ including schemes that iterate to self-consistency over the course of a time step from $t$ to $t+\Delta t$. However, in the present work we focus on the modified-midpoint algorithm, ${ }^{55}$ which corresponds to a propagator

$$
\mathbf{U}_{N}=\exp \left[-\mathrm{i}(\Delta t) \mathbf{F}_{N+1 / 2}\right]
$$


that updates the density matrix from $t_{N}$ to $t_{N+1}=t_{N}+$ $\Delta t$. The quantity $\mathbf{F}_{N+1 / 2}$ is the Fock matrix at time $t_{N}+\Delta t / 2$.

The time step $\Delta t$ used must be small enough to ensure numerical stability, and its value also determines the highest frequency-domain Fourier component that can be determined accurately, i.e., the Nyquist frequency $f_{\mathrm{Ny}}=\pi /(\Delta t)$ in atomic units. Previously, we found (as an empirical rule) that excitation energies are accurate up to somewhere between $f_{\mathrm{Ny}} / 5$ and $f_{\mathrm{Ny}} / 8$, depending on the functional that is used. ${ }^{39}$

2.1.2. Absorption Spectra. The absorption cross section $S(\omega)$ from a TDKS calculation is computed from the imaginary part of the trace of the frequencydependent polarizability tensor,

$$
S(\omega)=\left(\frac{4 \pi \omega}{3 c}\right) \mathfrak{I m}\left[\alpha_{x x}(\omega)+\alpha_{y y}(\omega)+\alpha_{z z}(\omega)\right]
$$

where

$$
\alpha_{\lambda \kappa}(\omega)=\frac{\partial \mu_{\lambda}(\omega)}{\partial \mathcal{E}_{\kappa}(\omega)}
$$

for $\lambda, \kappa \in\{x, y, z\}$. The quantity $\alpha_{\lambda \kappa}(\omega)$ describes the frequency-domain response of the dipole moment to an applied electric field $\mathcal{E}_{\kappa}(\omega)$ in the $\kappa$ direction. The frequency-dependent dipole moment may be calculated via discrete Fourier transform of the time-dependent dipole moment:

$$
\mu_{\lambda}(\omega)=\sum_{k=0}^{M} \mu_{\lambda}\left(t_{k}\right) e^{-\mathrm{i} \omega t_{k}}
$$

Here, $M$ is the total number of time steps and $t_{k}=k \Delta t$. In a TDKS calculation, one computes the time dependent density $\rho(\mathbf{r}, t)$, from which one can calculate $\mu_{\lambda}(t)$, leading ultimately to an absorption spectrum. The resolution of that absorption spectrum improves as the total simulation time $\left(t_{\max }=M \Delta t\right)$ increases.

2.1.3. Padé-Accelerated Transforms. For highenergy core excitation spectra a very small time step is required, making it computationally expensive to extend the simulations to the 20-30 fs of total propagation time that is often required to obtain a fully-converged spectrum. ${ }^{39}$ A solution is to use a Padé-accelerated Fourier transform, introduced in the present context by Lopata and co-workers, ${ }^{47}$ in which $\mu_{\lambda}(\omega)$ in eq. 8 is viewed as a polynomial expansion in $z=\exp (-\mathrm{i} \omega \Delta t)$ :

$$
\mu_{\lambda}(\omega)=\sum_{k=0}^{M} z^{k} \mu_{\lambda}\left(t_{k}\right)
$$

The Padé technique approximates a power series using rational functions whose numerator and denominator are both power series expansions of a certain order. Taking the polynomial order to be $P=M / 2$, eq. 9 is equivalent to

$$
\sum_{k=0}^{M} c_{k} z^{k} \sum_{m=0}^{P} b_{m} z^{m}=\sum_{k=0}^{P} a_{k} z^{k}
$$

By convention $b_{0}=1$, and thus eq. 10 consists of $2 P$ equations for a total of $2 P$ variables, $\left\{a_{i}\right\}$ and $\left\{b_{i}\right\}$, which are solved by separating the equations by orders in $z$. In matrix form, the solution for the $\left\{b_{i}\right\}$ coefficients is

$$
\mathbf{b}=\mathbf{G}^{-1} \mathbf{d}
$$

where $\mathbf{G}$ is a $P \times P$ matrix with $G_{k m}=c_{P-m+k}$, and $d_{k}=-c_{P+k}$. Coefficients $\left\{a_{i}\right\}$ are obtained from the $\left\{b_{i}\right\}$ :

$$
a_{k}=\sum_{m=0}^{k} b_{m} c_{k-m}
$$

Having $\mathbf{a}$ and $\mathbf{b}$, the Fourier transform of $\mu_{\lambda}(\omega)$ can be constructed, and the result is that a shorter time sequence of input data is required to obtain a converged Fourier transform. It should be noted that in contrast to the Padé-accelerated transform technique that was introduced in Ref. 47, which separately transforms the contribution to $\mu_{\lambda}(t)$ arising from fluctuations in each occupied-virtual function pair $\psi_{i}(\mathbf{r}, t) \psi_{a}(\mathbf{r}, t)$, the version that we have implemented transforms only the total dipole moment in eqs. 8 and 9 .

2.1.4. Complex Absorbing Potential. For energies as high as those encountered in XAS, the electronic transitions involve final states that are embedded in an ionization continuum. Lopata and co-workers have addressed this issue by ad hoc replacing any positive SCF eigenvalues, representing unbound states, with complex-valued eigenvalues in the MO representation of $\mathbf{P}(t),{ }^{42,43}$ which has the effect of giving those states finite lifetimes. Here, we pursue a different approach based on a real-space CAP.

Note that when atom-centered basis sets are used to represent $\mathbf{P}(t)$, as opposed to plane waves or real-space grids, the density matrix has compact support because the basis has finite extent. This leads to spurious reflection of the outgoing charge density at the "potential wall" that is created by the finite support of the basis set. These reflections give rise to fictitious peaks and other inaccuracies in the computed spectrum. To address this problem, certain calculations presented below use field amplitudes of $10^{-6}$ a.u., which is smaller than the values of $10^{-4}-10^{-5}$ a.u. that are more typical in TDKS calculations. However, the use of such a small excitation field leads to numerical issues in distinguishing the true fieldinduced density oscillations from numerical noise, since the magnitude of the oscillations is small compared to the 
numerical value of the initial-state density, $\rho(\mathbf{r}, 0)$. As such, calculations must be performed with tight convergence thresholds. The density $\rho(\mathbf{r}, 0)$ is only a stationary state up to the level of the SCF convergence threshold.

In order to avoid having small oscillations in $\rho(\mathbf{r}, t)$ be inundated by interference with the reflected wave, we introduce a CAP in order to absorb the outgoing wave. Our implementation is similar to that used by Schlegel and coworkers $^{51-54}$ to study strong-field ionization dynamics and is constructed from a set of overlapping, atomcentered spherical potential functions. Each potential is zero within the cutoff radius $\left(r_{0}\right)$ of the nucleus on which it is centered, then rises quadratically with curvature $\eta$. The CAP is therefore constructed from a set of atomcentered functions

$$
f_{k}^{\mathrm{CAP}}(\mathbf{r})= \begin{cases}0, & \left\|\mathbf{r}-\mathbf{R}_{k}\right\|<r_{0} \\ \eta\left\|\mathbf{r}-\mathbf{R}_{k}\right\|^{2}, & \left\|\mathbf{r}-\mathbf{R}_{k}\right\| \geq r_{0}\end{cases}
$$

for $k=1,2, \ldots, N_{\mathrm{A}}$, where $\mathbf{R}_{k}$ indicates the location of the $k$ th nucleus and $N_{\mathrm{A}}$ is the number of atoms. The value of the CAP itself at a given point $\mathbf{r}$ is taken to be the minimum value of all the functions $f_{k}^{\mathrm{CAP}}(\mathbf{r})$, with the additional stipulation that the potential is cut off at a maximum value $E_{\max }=10 E_{h}$ in order to avoid numerical overflow problems. Thus, the real-space CAP function is defined as

$$
v_{\mathrm{CAP}}(\mathbf{r})=\min \left\{E_{\max }, f_{1}^{\mathrm{CAP}}(\mathbf{r}), \ldots, f_{N_{\mathrm{A}}}^{\mathrm{CAP}}(\mathbf{r})\right\} .
$$

In practice, we require matrix representations of the real-space potential function $v_{\mathrm{CAP}}(\mathbf{r})$. In the $\mathrm{AO}$ basis, consisting of atom-centered Gaussian functions $\left\{g_{\mu}(\mathbf{r})\right\}$, this representation is

$$
V_{\mu \nu}^{\mathrm{CAP}}=\int g_{\mu}(\mathbf{r}) v_{\mathrm{CAP}}(\mathbf{r}) g_{\nu}(\mathbf{r}) d \mathbf{r} .
$$

The integral is evaluated by numerical quadrature, using the DFT quadrature grid. ${ }^{56}$ The matrix $\mathbf{V}^{\mathrm{CAP}}$ does not depend on time and can be constructed once, at the beginning of a TDKS simulation, and is then added to the Fock matrix at each time step:

$$
\mathbf{F}^{\mathrm{CAP}}(t)=\mathbf{F}(t)-\mathrm{i} \mathbf{V}^{\mathrm{CAP}} .
$$

Note that $\mathbf{V}^{\mathrm{CAP}}$ is a symmetric matrix and therefore $-\mathrm{i} \mathbf{V}^{\mathrm{CAP}}$ is skew-Hermitian, meaning that $\mathbf{F}^{\mathrm{CAP}}$ is not Hermitian despite the fact that $\mathbf{F}$ is symmetric. This requires modification to the usual propagator, which is accomplished by factorizing the relevant exponential using the splitting technique:

$$
\begin{aligned}
\exp \left[-\mathrm{i}(\Delta t) \mathbf{F}^{\mathrm{CAP}}\right] \\
=\exp \left[-\mathrm{i}(\Delta t)\left(\mathbf{F}-\mathrm{i} \mathbf{V}^{\mathrm{CAP}}\right)\right] \\
=\exp \left[-(\Delta t / 2) \mathbf{V}^{\mathrm{CAP}}\right] \exp [-\mathrm{i}(\Delta t) \mathbf{F}] \\
\quad \times \exp \left[-(\Delta t / 2) \mathbf{V}^{\mathrm{CAP}}\right] .
\end{aligned}
$$

In this factorization, the leading and trailing exponentials involve symmetric ones and the middle exponential involves a skew-Hermitian matrix, and each of these can be easily diagonalized to evaluate the corresponding exponential function.

2.2. LR-TDDFT. The LR-TDDFT approach, which is often simply called "TDDFT", ${ }^{28}$ corresponds to taking the linear response of eq. 3 to a weak perturbation. ${ }^{27}$ As such, LR-TDDFT can be regarded as the "weak-field limit" of TDDFT, and identical spectra are obtained with the TDKS approach when the perturbing field is small and the time-dependent simulation is propagated for a sufficiently long time. ${ }^{38,39}$

For completeness, we recapitulate the LR-TDDFT eigenvalue equation for excitation energies $\omega:^{28}$

$$
\left(\begin{array}{ll}
\mathbf{A} & \mathbf{B} \\
\mathbf{B}^{\dagger} & \mathbf{A}^{\dagger}
\end{array}\right)\left(\begin{array}{l}
\mathbf{x} \\
\mathbf{y}
\end{array}\right)=\omega\left(\begin{array}{rr}
-\mathbf{1} & \mathbf{0} \\
\mathbf{0} & \mathbf{1}
\end{array}\right)\left(\begin{array}{l}
\mathbf{x} \\
\mathbf{y}
\end{array}\right)
$$

The orbital Hessian matrices $\mathbf{A}$ and $\mathbf{B}$ are given by

$$
\begin{aligned}
A_{i a, j b} & =\left(\varepsilon_{a}-\varepsilon_{i}\right) \delta_{i j} \delta_{a b}+\frac{\partial F_{i a}}{\partial P_{j b}} \\
B_{i a, j b} & =\frac{\partial F_{i a}}{\partial P_{b j}}
\end{aligned}
$$

where $i, j$ index occupied MOs and $a, b$ index virtual MOs. The Tamm-Dancoff approximation (TDA) simplifies eq. 18 by neglecting the de-excitation amplitudes $\mathbf{y}$, which are often $\sim 100 \times$ smaller than the largest $\mathbf{x}$ amplitudes. The resulting TDA eigenvalue equation is

$$
\mathbf{A x}=\omega \mathbf{x} .
$$

This LR-TDDFT formalism has been adapted for the calculation of core excitation spectra using frozen occupied orbitals, ${ }^{6,33,57-59}$ i.e., core/valence separation. We will compare this LR-TDDFT approach to the TKDS approach, for benchmark purposes and to highlight advantages of the latter formalism.

\section{Computational Methods}

Results presented here based on a completely new implementation of the TDKS model in Q-Chem v. 5.4, ${ }^{7}$ which replaces the original module written by Nguyen and Parkhill. ${ }^{60,61}$ In the new module, time propagation can be accomplished either based on the modifiedmidpoint algorithm ${ }^{55}$ or else self-consistent predictorcorrector algorithms. ${ }^{39}$ The predictor-corrector algorithms allow for stable simulations using much larger time steps, although the primary limitation on $\Delta t$ in the present work is the Nyquist frequency $f_{\mathrm{Ny}}=\pi /(\Delta t)$ that sets the maximum excitation energy that can reliably be obtained from a TDKS simulation. The requisite energy scales for x-ray spectroscopy are quite high and thus $\Delta t$ must be small, and for that reason all of the calculations reported here use the modified-midpoint propagator. Additional features needed for XAS, as described 
above, have been implemented in Q-Chem for the first time as part of this work.

3.1. Functionals and Numerical Parameters. We set $\Delta t=0.02 \mathrm{a} . \mathrm{u}$. for all calculations at the oxygen and carbon K-edges, and $\Delta t=0.004$ a.u. for the sulfur $\mathrm{K}$ edge. Our most conservative previous estimate is that TDKS spectra are valid up to energies corresponding to $f_{\mathrm{Ny}} / 8 .^{39}$ Recalling that $f_{\mathrm{Ny}}=\pi /(\Delta t)$ in atomic units, this puts the upper bound at $534 \mathrm{eV}$ for the larger time step and $2670 \mathrm{eV}$ for the smaller one, which are the energy ranges that correspond to the oxygen and sulfur Kedges, respectively. The method of Padé approximants, ${ }^{47}$ as described above, is applied to post-process all of the time-dependent dipole moment data obtained from the TDKS simulations, which accelerates convergence of the spectrum and makes it possible to obtain good results with short simulations. In our experience, simulation times $\gtrsim 30 \mathrm{fs}$ are required to obtain a fully converged spectrum in the absence of acceleration techniques, ${ }^{39}$ whereas the Padé approach is able to resolve rough spectra in as little as 3-5 fs of simulation time and wellconverged spectra in $<10 \mathrm{fs}$. Spectra reported here are based on at least $5 \mathrm{fs}$ of total propagation time and their convergence has been checked against longer simulations.

In simulations that use a CAP, the turn-on radius in eq. 13 is set to $r_{0}=9.5 \mathrm{bohr}$ and the curvature to $\eta=$ 4 a.u.. Note that for a Gaussian of the form $g(r)=$ $\exp \left(-\zeta r^{2}\right)$, the full width at half maximum (FWHM) is $^{48}$

$$
\operatorname{FWHM}(\zeta)=\frac{2 \sqrt{\ln 2}}{\zeta^{1 / 2}}
$$

and the $2 \sigma$ radial extent of $g(r)$ is $(2 / \zeta)^{1 / 2}$. As an example, the most diffuse exponents for carbon and oxygen in the def2-TZVPD basis set are both $\zeta \approx 0.05 \mathrm{bohr}^{-1}$, corresponding to a $2 \sigma$ radial extent of 6.3 bohr. As such, the CAP affects only the outermost tail of the density.

Starting from a ground-state SCF calculation, which is converged to a threshold of $10^{-8} E_{h}$ in the present work, the density is perturbed by means of a $\delta$-function pulse in which the external field is only nonzero during the first two steps. To normalize the initial perturbation across different choices of $\Delta t$, we report the integrated field strength $\overline{\mathcal{E}}$ whose components are

$$
\overline{\mathcal{E}}_{\kappa}=\mathcal{E}_{\kappa} \Delta t
$$

for $\kappa \in\{x, y, z\}$. (The actual field amplitude, in the sense of eq. 7 , is therefore $\mathcal{E}_{\kappa}=\overline{\mathcal{E}}_{\kappa} / \Delta t$. However, that quantity is not needed to compute the absorption spectrum, which instead comes from the Fourier transform of the fluctuating dipole moment function.) The perturbing field consists of equal components in each Cartesian direction $\left(\overline{\mathcal{E}}_{x}=\overline{\mathcal{E}}_{y}=\overline{\mathcal{E}}_{z}\right)$, which ensures that the perturbation creates a superposition of all excited states, regardless of electronic symmetry. Unless otherwise specified, each of these components is set to $\overline{\mathcal{E}}_{\kappa}=10^{-6}$ a.u., although we also report some calculations with stronger fields.

Some basis-set testing is presented in Section 3 3.2. For density functionals, we use the "short-range corrected" (SRC) functionals SRC1 and SRC2. ${ }^{62,63}$ These are rangeseparated hybrid functionals based on the short-range exchange functional $\mu \mathrm{BLYP},{ }^{64,65}$ which were parameterized specifically for $\mathrm{x}$-ray spectroscopy at the K-edge. ${ }^{62,63}$ These functionals use a large fraction of Hartree-Fock exchange attenuated on a length scale of $<1 \AA{ }^{62}$ which presumably corrects for differential self-interaction error between the core and valence virtual orbitals. Actually there are two versions of both functionals, SRCn$\mathrm{r} 1$ and $\mathrm{SRC} n-\mathrm{r} 2,{ }^{5}$ where the former are parameterized for "first-row" elements (C, N, O, etc.) and contains $50 \%$ (SRC1) and 55\% (SRC2) short-range Hartree-Fock exchange, whereas the "second-row" versions (SRCnr2) contain $87 \%$ (SRC1) and 91\% (SRC2) short-range Hartree-Fock exchange.

3.2. Tests of Basis Set and Active Spaces. Several previous studies have explored modified Gaussian basis sets for use in XAS calculations. ${ }^{66-70}$ In some cases, standard Gaussian basis sets have been "uncontracted", ${ }^{66-68}$ meaning that each Gaussian primitive is used as an independent basis function, in order to improve the variational flexibility of the core functions, which are usually highly contracted. A separate strategy $^{68-70}$ is to use basis sets that include core-valence polarization functions, such as cc-pCVXZ. ${ }^{71}$ It has been suggested that the latter basis sets work well for corelevel spectroscopy precisely because the additional corevalence polarization functions are uncontracted. ${ }^{69}$

In contrast to the behavior observed with correlated wave functions, ${ }^{67,70}$ our testing with LR-TDDFT reveals that uncontracting the basis sets modifies the excitation energies by only $\sim 0.1 \mathrm{eV}$; see Figs. S1 and S2. We examined oxygen K-edge excitations in formaldehyde, acetone, uracil, and methionine, and found this to be true for each of the first $25 \mathrm{O}(1 \mathrm{~s}) \rightarrow$ virtual transitions, in a variety of basis sets including 6-311++ $\mathrm{G}^{* *}, 6-311(2+, 2+) \mathrm{G}^{* *}$, (aug-)cc-pVTZ, (aug-)cc-pCVTZ, and def2-TZVVPD. These 25 states span a range of up to $45 \mathrm{eV}$ in some cases. Comparing cc-pVTZ to cc-pCVTZ in the case of acetone, the excitation energy for the first transition $[\mathrm{O}(1 \mathrm{~s}) \rightarrow$ LUMO] at $531.4 \mathrm{eV}$ changes by $<0.1 \mathrm{eV}$, while that for the 25 th excited state (at $549.6 \mathrm{eV}$ ) changes by $0.7 \mathrm{eV}$. A previous LR-TDDFT study also concluded that basis set effects are rather small for XAS calculations, although somewhat more pronounced for x-ray emission. ${ }^{68}$ (Emission is not considered here.) A summary of basis set effects is presented in Table S1, which also demonstrate that the SRC functionals predict oxygen and carbon Kedge excitation energies within $0.1-0.2 \mathrm{eV}$ for the aforementioned molecules, without any relativistic correction.

The LR-TDDFT calculations described above were performed using a frozen occupied orbital approximation 


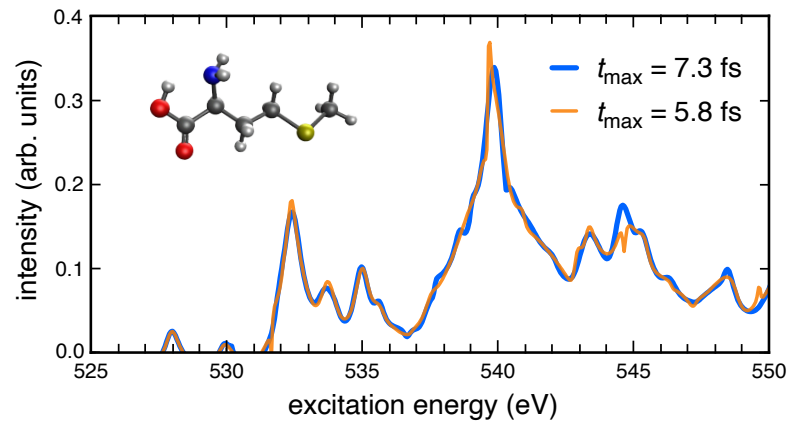

Fig. 1. XAS spectra of methionine at the oxygen K-edge, obtained from TDKS simulations with Padé-accelerated Fourier transforms at the SRC1-r1/def2-TZVPD level, starting from a $\delta$-function perturbation with $\overline{\mathcal{E}}_{x}=\overline{\mathcal{E}}_{y}=\overline{\mathcal{E}}_{z}=10^{-4}$ a.u.. The two spectra differ only in the total length of simulation time, as indicated in the legend, and the close agreement suggests that the simulation with $t_{\max }=5.8 \mathrm{fs}$ is converged.

in which in the only active occupied MOs are the 1s orbitals of the atom in question. ${ }^{57}$ (For carbon or oxygen K-edge XAS, this means the 1s orbitals for all of the carbon atoms or all of the oxygen atoms, respectively.) Fig. S1d compares the oxygen K-edge LR-TDDFT spectrum for methionine using this reduced excitation space, versus the one that is computed using the full excitation space. This results in very little change to the spectrum, consistent with LR-TDDFT results reported by Besley and co-workers, ${ }^{57,59}$ and with the general validity of core/valence separation for K-edge excitations. ${ }^{34}$ The TDA (eq. 20) also results in negligible changes to the spectrum (Fig. S1e) and is used in all subsequent LR-TDDFT calculations.

\section{Results and Discussion}

4.1. Evaluation of TDKS Protocols. We first demonstrate the utility of Padé-accelerated transforms to dramatically reduce the total simulation time required to obtain a converged spectrum. To check convergence with respect to total simulation time, we calculate the XAS spectrum of the methionine molecule at the oxygen K-edge. Two such spectra are plotted in Fig. 1, both of which make use of Padé approximants but corresponding to different simulation lengths, $t_{\max }=5.8 \mathrm{fs}$ versus $t_{\max }=7.3 \mathrm{fs}$. The two spectra are in very good agreement, suggesting convergence in $<6 \mathrm{fs}$ of simulation time, which is considerably smaller than what is required in the absence of Padé approximants.

We next demonstrate the use of the CAP. Figure 2 displays the same oxygen K-edge spectrum for methionine, but computed with a larger $\delta$-function pulse $\left(\overline{\mathcal{E}}_{x}=\overline{\mathcal{E}}_{y}=\right.$ $\overline{\mathcal{E}}_{z}=10^{-4}$ a.u., as compared to $\overline{\mathcal{E}}_{x}=\overline{\mathcal{E}}_{y}=\overline{\mathcal{E}}_{z}=10^{-6}$ a.u. in Fig. 1), and in the present case computed either with or without a CAP. Some small variations in the relative

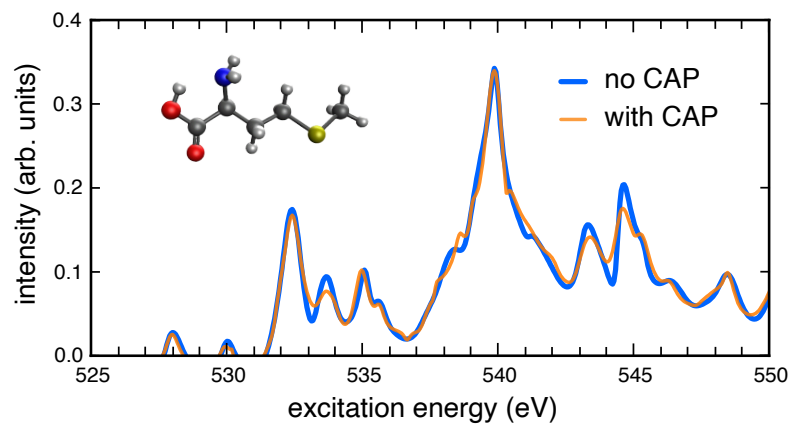

Fig. 2. XAS spectra of methionine at the oxygen Kedge, computed from TDKS simulations at the SRC1-r1/def2TZVPD level with and without a CAP. Both TDKS calculations were propagated for 7.3 fs following a $\delta$-function pulse of magnitude $\overline{\mathcal{E}}_{x}=\overline{\mathcal{E}}_{y}=\overline{\mathcal{E}}_{z}=10^{-4}$ a.u..

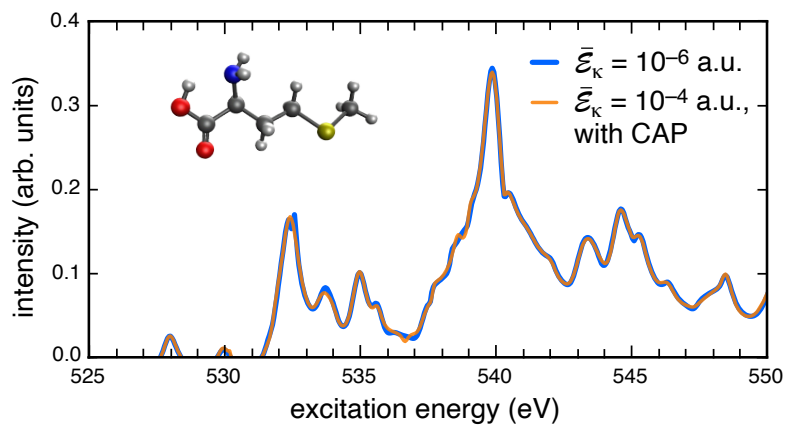

Fig. 3. XAS spectra of methionine at the oxygen Kedge, computed from TDKS simulations at the SRC1-r1/def2TZVPD level starting from a $\delta$-function pulse as indicated. (The larger field strength uses a CAP but the smaller field strength does not.) Both TDKS calculations were propagated for $7.3 \mathrm{fs}$.

intensities of various features are evident, when comparing the spectra with and without the CAP, but mostly the spectra are quite similar.

This comparison serves as a baseline for the comparison that is shown in Fig. 3, in which the same spectrum for methionine is computed with field strengths of $\overline{\mathcal{E}}_{x}=\overline{\mathcal{E}}_{y}=\overline{\mathcal{E}}_{z}=10^{-4}$ a.u. and $\overline{\mathcal{E}}_{x}=\overline{\mathcal{E}}_{y}=\overline{\mathcal{E}}_{z}=10^{-6}$ a.u. in the presence or absence of a CAP, respectively. (The spectrum computed with the smaller impulse field, with a CAP, is common to both Figs. 2 and 3.) The two spectra in Fig. 3 are nearly identical and much more similar to one another as compared to the two spectra shown in Fig. 2, demonstrating that the CAP-free spectrum converges (in the limit of a weak impulse) to the same spectrum that is obtained using a stronger field, augmented with a CAP. This suggests that the CAP is fulfilling its role, eliminating artifacts due to reflection of the outgoing wave and thus allowing larger field strengths to be used. Larger field strengths mean less numerical noise. It is worth noting that the use of a real-space CAP is computationally straightforward and does not require $a$ 


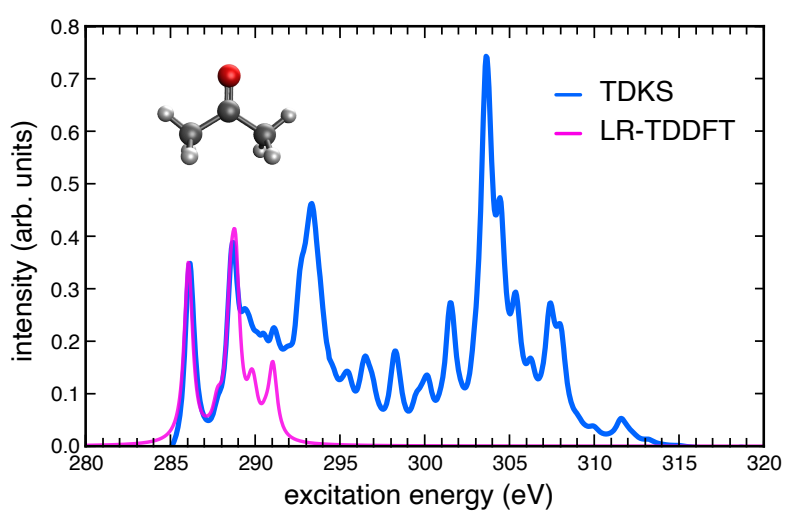

Fig. 4. XAS spectra of acetone at the carbon K-edge, computed at the SRC1-r1/6-311 $(2+, 2+) \mathrm{G}^{* *}$ level using either the TDKS approach or else LR-TDDFT. The LR-TDDFT spectrum consists of 25 roots and uses an active space consisting of the $\mathrm{C}(1 \mathrm{~s})$ occupied MOs along with all of the virtual MOs, and each excitation energy broadened using a Lorentzian function with a width parameter $\Gamma=0.6 \mathrm{eV}$. The TDKS calculation was propagated for $7.3 \mathrm{fs}$ but the spectrum is indistinguishable from that obtained using 14.4 fs of propagation time.

priori determination of vacuum energy cutoffs, as needed for the MO-based absorbing potential that has been employed by Lopata and co-workers ${ }^{42,43}$ which modifies the SCF eigenvalues rather than adding an explicit potential.

4.2. Comparison to LR-TDDFT. We next compare the spectra obtained with the TDKS approach to more traditional LR-TDDFT calculations, using carbon K-edge XAS of acetone as a first example (Fig. 4). The LR-TDDFT result is obtained by a restricted set of single excitations in which the active space consists of the complete set of virtual orbitals, along with the three $\mathrm{C}(1 \mathrm{~s})$ orbitals from the occupied space. The first 25 LR-TDDFT states are computed and the resulting stick spectrum is broadened using a Lorentzian function whose width parameter is selected to match the width of the first feature in the TDKS spectrum, around $286 \mathrm{eV}$ in Fig. 4. The intensity units are arbitrary and the spectra have been scaled so that their intensities are the same for that first peak. The LR-TDDFT spectrum then provides a nearly quantitative match to the TDKS spectrum through the second peak, at $288.5 \mathrm{eV}$, although 25 excited states proves to be insufficient to go beyond the second peak in the state-by-state LR-TDDFT approach. Two minor peaks at 289.5 and $291.0 \mathrm{eV}$, in between the second and third major peaks, are clearly evident in the LR-TDDFT spectrum but with too little intensity, and above that the intensity quickly falls off to zero as the calculation reaches the requested number of states. Note that the TDKS spectrum is valid in this case across the entire energy range of $\mathrm{C}(1 \mathrm{~s}) \rightarrow$ virtual transitions, because the time step (and therefore the Nyquist frequency) was set based on the higher-energy oxygen K-edge, and the lower-

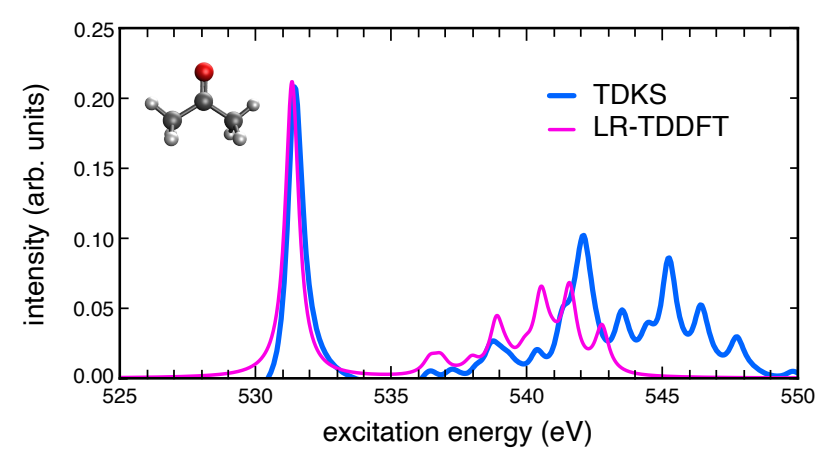

Fig. 5. XAS spectra of acetone at the oxygen K-edge, computed at the SRC1-r1/6-311 $(2+, 2+) \mathrm{G}^{* *}$ level using either the TDKS approach or else LR-TDDFT. The LR-TDDFT spectrum consists of 25 roots and uses an active space consisting of the $\mathrm{O}(1 \mathrm{~s})$ occupied MOs along with all of the virtual MOs, and each excitation energy broadened using a Lorentzian function with a width parameter $\Gamma=0.6 \mathrm{eV}$. The TDKS calculation was propagated for $7.3 \mathrm{fs}$ but the spectrum is indistinguishable from that obtained using $14.4 \mathrm{fs}$ of propagation time.

energy carbon K-edge spectrum is a by-product of the same calculation. In contrast, the 25 states computed in the LR-TDDFT calculation span a range of only about $6 \mathrm{eV}$, from $286-291 \mathrm{eV}$.

The analogous comparison between LR-TDDFT and TDKS at the oxygen K-edge is presented in Fig. 5, again for the acetone molecule. It should be noted that the TDKS spectrum here comes from the same simulation that was used to obtain the carbon K-edge spectrum in Fig. 4, whereas the LR-TDDFT approach requires a separate calculation at either edge, with different truncations of the active space. With only the $\mathrm{O}(1 \mathrm{~s})$ orbitals in the occupied part of the active space, the first 25 roots span an energy from from 531-543 eV. This LR-TDDFT calculation matches the first peak in the TDKS spectrum, which is isolated from the rest of the spectrum, and the energies of the second and third peaks also match fairly well although there are discrepancies in the relative intensities of the higher-energy features. Above $540 \mathrm{eV}$, the two spectra are clearly different.

Having compared the spectra for acetone obtained with TDKS and LR-TDDFT at a single level of theory, we next extend this analysis to compare two different molecules (acetone and formaldehyde), two different functionals (SRC1-r1 and SRC2-r1), and two different basis sets $\left[6-311(2+, 2+) \mathrm{G}^{* *}\right.$ and cc-pCVDZ]. Table 1 lists the lowest excitation energies obtained from LRTDDFT, using the usual $\mathrm{O}(1 \mathrm{~s})$ and $\mathrm{C}(1 \mathrm{~s})$ active-space approximation for the oxygen and carbon K-edges, and compares these values to the corresponding peak in the TDKS spectrum. Differences are $\lesssim 0.1 \mathrm{eV}$ with a maximum deviation of $0.14 \mathrm{eV}$, demonstrating consistency in the first peak positions between TDKS and LR-TDDFT as applied to K-edge states. From another point of view, this comparison (and the ones presented Figs. 4 and 5) 
Table 1. Comparison of Carbon and Oxygen K-Edge Peaks Obtained with TDKS Versus LR-TDDFT.

\begin{tabular}{|c|c|c|c|c|c|}
\hline \multirow{2}{*}{ Functional } & \multirow{2}{*}{ Basis Set } & \multicolumn{2}{|c|}{$\left(\mathrm{CH}_{3}\right)_{2} \mathrm{CO}$} & \multicolumn{2}{|c|}{$\mathrm{H}_{2} \mathrm{CO}$} \\
\hline & & $\Delta E^{a}$ & deviation $^{b}$ & $\Delta E^{a}$ & deviation $^{b}$ \\
\hline SRC1-r1 & $6-311(2+, 2+) \mathrm{G}^{* *}$ & 286.07 & +0.09 & 285.62 & +0.05 \\
\hline SRC2-r1 & $6-311(2+, 2+) \mathrm{G}^{* *}$ & 286.59 & +0.05 & 286.09 & +0.04 \\
\hline SRC1-r1 & cc-pCVDZ & 286.40 & +0.08 & 286.09 & -0.10 \\
\hline SRC1-r1 & $6-311(2+, 2+) \mathrm{G}^{* *}$ & 531.35 & +0.09 & 530.92 & +0.14 \\
\hline SRC2-r1 & $6-311(2+, 2+) \mathrm{G}^{* *}$ & 531.08 & +0.09 & 530.82 & +0.07 \\
\hline SRC1-r1 & cc-pCVDZ & 531.54 & +0.12 & 531.14 & +0.03 \\
\hline
\end{tabular}

${ }^{a}$ Lowest LR-TDDFT excitation energy, with an occupied active space that includes only the $\mathrm{C}(1 \mathrm{~s})$ or $\mathrm{O}(1 \mathrm{~s})$ orbitals. ${ }^{b} \Delta E(\mathrm{TDKS})-\Delta E(\mathrm{LR}-\mathrm{TDDFT})$

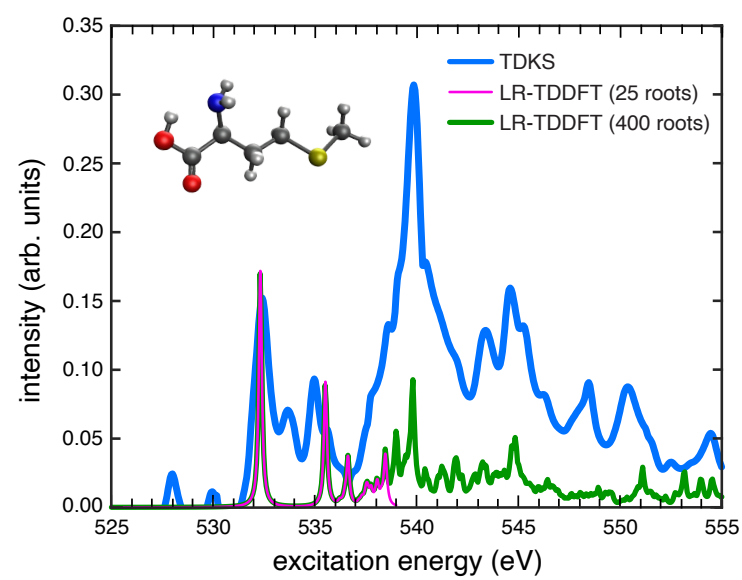

Fig. 6. XAS spectra of methionine at the oxygen K-edge, computed at the SRC1-r1/def2-TZVPD level from both a TDKS simulation and from either of two LR-TDDFT calculations. The latter employ an active space consisting of the $\mathrm{O}(1 \mathrm{~s})$ occupied MOs and all of the virtual MOs, but a different number of eigenvalues (either 25 or 400) is computed. In either case, the stick spectra are broadened using a Lorentzian function with a width $\Gamma=0.18 \mathrm{eV}$. The TDKS spectrum is computed (using a CAP) based on $7.3 \mathrm{fs}$ of total propagation time, following a $\delta$-function impulse with $\overline{\mathcal{E}}_{x}=\overline{\mathcal{E}}_{y}=\overline{\mathcal{E}}_{z}=10^{-4}$ a.u. As discussed in the text, features below $531 \mathrm{eV}$ correspond to $\mathrm{N}(1 \mathrm{~s}) \rightarrow$ continuum transitions, whereas the oxygen K-edge begins above $531 \mathrm{eV}$. Figure is adapted from Ref. 7; copyright 2021 American Institute of Physics.

confirm the accuracy of the core/valence separation approximation for K-edge excitations. ${ }^{34}$

Finally, we compare TDKS and LR-TDDFT spectra for methionine at the oxygen K-edge, in Fig. 6. Two LRTDDFT spectra are shown, consisting of either 25 or 400 roots with the usual K-edge truncation of the occupied space in both cases, meaning that only $\mathrm{O}(1 \mathrm{~s})$ orbitals are included from the occupied space. Two pre-edge features (one at $530 \mathrm{eV}$ and another just below that) are missing from the LR-TDDFT spectrum, indicating that these do not originate from $\mathrm{O}(1 \mathrm{~s})$ orbitals. These features correspond to excitations from $\mathrm{N}(1 \mathrm{~s})$ orbitals to the very highest-energy virtual MOs, that latter of which are "or- thogonalized discretized continuum" states. ${ }^{48}$ This becomes clear if one computes all $o \times v$ eigenvalues of the LR-TDDFT eigenvalue problem in eq. 20, which is feasible in smaller basis sets. As shown in Fig. S3, when the full set of LR-TDDFT eigenvalues is examined, there is a semi-continuous sequence of dark states that connects each of the x-ray K-edges (carbon, nitrogen, oxygen, ...), representing core $\rightarrow$ continuum excitations. These are called "intruder states" in Ref. 47, although this risks confusion with (unrelated) terminology in multireference perturbation theory. ${ }^{72,73}$ These ionization contributions are eliminated in Ref. 47 by transforming $\mathbf{P}(t)$ into the $\mathrm{MO}$ basis and removing individual contribution $P_{i a}(t)$ corresponding to the undesirable (discretized continuum) orbitals $\psi_{a}$. We have not done so here, as it is easy to recognize the that oxygen K-edge spectrum in Fig. 6 begins with the feature at $532.5 \mathrm{eV}$.

Regarding that feature, the oxygen K-edge at $532.5 \mathrm{eV}$ appears at the same energy in all three spectra in Fig. 6. However, the LR-TDDFT oscillator strength drops off precipitously at higher energies and subsequent features exhibit discrepancies with respect to the TDKS calculation, in the range of $533-535 \mathrm{eV}$, which persist even when 400 excited states are included in the LR-TDDFT calculation. Tests of the effect of restricting the LRTDDFT excitation manifold (Fig. S1 of this work and also Ref. 57), as well as the accuracy of the core/valence approximation for K-edge excitations more generally, ${ }^{34}$ suggest that errors arising from frozen occupied orbitals cannot account for the observed discrepancies. At the same time, convergence tests of the TDKS approach (Section 44.1) suggest that the real-time spectrum is converged.

4.3. Case Study: Sulfur K-edge of a Copper Dithiolene Complex Lastly we present an exemplary application, computing XAS at the sulfur Kedge for oxidized and reduced forms of a copper bis(maleonitriledithiolate) complex, $\left[\mathrm{Cu}(\mathrm{mnt})_{2}\right]^{1-/ 2-}$, where $\mathrm{mnt}=1,2$-dicyanoethylene-1,2-dithiolate (inset of Fig. 7). The (mnt) ${ }^{2-}$ moiety is one of the simplest examples of a dithiolene ligand and a prototypical noninnocent ligand in transition metal chemistry. ${ }^{74}$ Metal dithiolene 


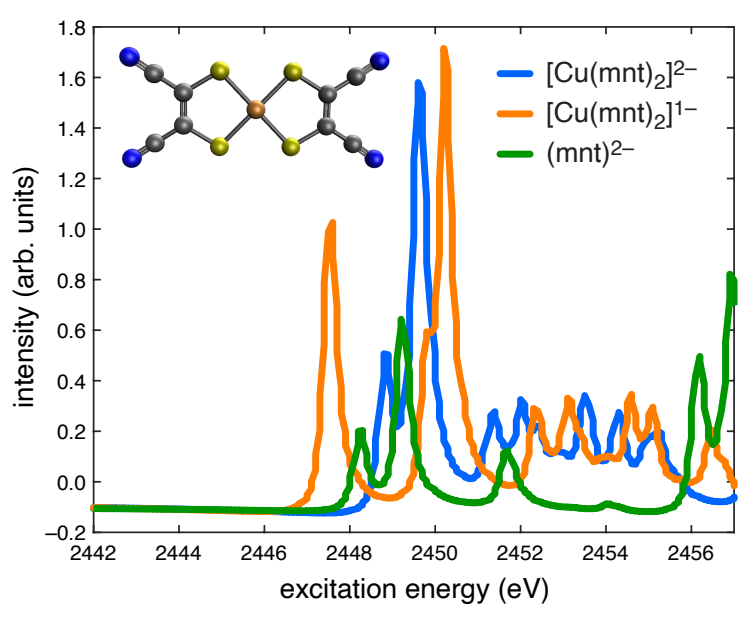

Fig. 7. XAS at the sulfur K-edge, computed for a Cu-thiolene complex whose structure is shown, computed using TDKS $\left(\Delta t=0.004\right.$ a.u. and $\overline{\mathcal{E}}_{x}=\overline{\mathcal{E}}_{y}=\overline{\mathcal{E}}_{z}=10^{-4}$ a.u., with $14.7 \mathrm{fs}$ of total propagation time) at the SRC1-r1/6-31G* level. The metal complex is $\left[\mathrm{Cu}(\mathrm{mnt})_{2}\right]^{1-/ 2-}$ in either of two oxidation states, and the spectrum of the free $(\mathrm{mnt})^{2-}$ ligand is also shown for comparison.

complexes display an intense pre-edge feature typical of ligand K-edges in the spectroscopy of transition metal complexes, where both the position and the integrated intensity of the pre-edge feature are sensitive to the oxidation state of the metal. Any change in the electronic structure of the complex is thus expected to manifest in sulfur K-edge XAS.

That spectrum has been computed using the TDKS approach for both $\left[\mathrm{Cu}(\mathrm{mnt})_{2}\right]^{1-/ 2-}$ oxidation states as well as for the free $(\mathrm{mnt})^{2-}$ ligand, with results shown in Fig. 7. The sulfur K-edge is a higher-energy regime as compared to the second-row elements examined above and these calculations therefore use a smaller time step, $\Delta t=0.004$ a.u., following a $\delta$-function perturbation with $\overline{\mathcal{E}}_{x}=\overline{\mathcal{E}}_{y}=\overline{\mathcal{E}}_{z}=10^{-4}$ a.u.

For the $\mathrm{Cu}(\mathrm{II})$ and $\mathrm{Cu}(\mathrm{III})$ complexes, we find the first peak appears at $2448.8 \mathrm{eV}$ and $2447.5 \mathrm{eV}$, respectively. This feature corresponds to a $\mathrm{S}(1 \mathrm{~s}) \rightarrow \mathrm{Cu}(3 \mathrm{~d})$ transition and the higher oxidation state, $\mathrm{Cu}(\mathrm{III})$, experiences increased charge donation from the sulfur center, leading to a larger intensity as compared to the same transition in the reduced form of the complex. Because this ligand-tometal charge-transfer transition is absent in the spectrum of the free ligand, the lowest-energy peaks in the spectrum of free $(\mathrm{mnt})^{2-}$ correspond to overlapping ligandcentered $\mathrm{S}(1 \mathrm{~s}) \rightarrow \pi^{*}$ transitions. The analogous transitions in the $\mathrm{Cu}(\mathrm{II})$ complex can be observed at $2449.3 \mathrm{eV}$ and in the $\mathrm{Cu}(\mathrm{III})$ complex at $2450.1 \mathrm{eV}$, blue-shifted relative to the free ligand. A similar blue-shift has been observed experimentally, ${ }^{75}$ where it was rationalized in terms of stabilization of the $\mathrm{S}(1 \mathrm{~s})$ orbitals in the bound ligands relative to the free ligand, with an increase in positive charge on the sulfur center.
TDKS calculations therefore reproduce the observed spectroscopic effect of a change in oxidation state on the $\mathrm{Cu}$ center, as it manifests in the intensity of ligand-tometal transitions at the sulfur K-edge, as well as the blue-shifting of the ligand-centered edge transitions. It should be noted that with a time step (and corresponding Nyquist frequency) suitable to obtain the sulfur K-edge, one obtains from the TDKS simulation all of the lowerenergy K-edge spectra as well, including carbon, nitrogen, and oxygen in the present example. This demonstrates the practical utility of the TDKS approach, as these would be separate calculations with LR-TDDFT. In addition, we have seen that several hundred excited states is only sufficient to compute the first $3-5 \mathrm{eV}$ of a typical broadband K-edge spectrum, a problem that will grow worse in solid-state semiconductor materials with a denser manifold of bound virtual levels.

\section{Conclusions}

This work describes an implementation of TDKS or "real-time" TDDFT and explores its application to corelevel XAS at the K-edge of various main-group elements. The TDKS approach allows for all of the relevant K-edges to be computed at once in a single broadband calculation, up to an energy scale that varies inversely with the time step that is used to integrate the equations of motion. The more traditional LR-TDDFT approach requires that such spectra be computed state-by-state, and hundreds of states must be computed to extend the spectrum 3-5 eV beyond the lowest transition (1s $\rightarrow$ LUMO). By employing the method of Padé approximants to accelerate convergence of the Fourier transform of the fluctuating dipole moment, and a novel use of absorbing potentials that helps to remove artifacts caused by reflection of the outgoing wave at larger field strengths, we are able to obtain resolved broadband spectra in as little as $3-5$ fs of simulation time and fully-converged spectra in $<10 \mathrm{fs}$, significantly shorter than the $20-30$ fs that is usually required.

We document that the dependence on the Gaussian basis set is not nearly so severe as what has been reported in the literature for other quantum chemistry approaches aimed at core-level spectroscopy. For TDKS, standard Gaussian basis sets that are common for ground-state DFT can be used without modification. All of the relevant K-edges (carbon, nitrogen, oxygen, ...) up to the highest-energy one are obtained in a single calculation, since it is the highest-energy part of the spectrum that dictates the time step that is required for the simulations. Together, these features make TDKS a powerful tool for x-ray spectroscopy, especially for solid-state semiconductor materials with a potentially dense manifold of bound virtual levels, for which the state-by-state nature of the LR-TDDFT approach will become prohibitively expensive. 


\section{Acknowledgements}

This work was supported by National Science Foundation grant nos. CHE-1665322 and CHE-1955282. Calcu- lations were performed at the Ohio Supercomputer Center under project no. PAA0003. ${ }^{76}$
1 P. Norman and A. Dreuw, "Simulating x-ray spectroscopies and calculating core-excited states of molecules", Chem. Rev., 118, 7208-7248 (2018).

2 S. I. Bokarev and O. Kühn, "Theoretical x-ray spectroscopy of transition metal compounds", WIREs Comput. Mol. Sci., 10, e1433 (2020).

3 J. M. Kasper, T. F. Stetina, A. J. Jenkins, and X. Li, " $A b$ initio methods for L-edge x-ray absorption spectroscopy", Chem. Phys. Rev., 1, 011304 (2020).

${ }^{4}$ C. D. Rankine and T. J. Penfold, "Progress in the theory of x-ray spectroscopy: From quantum chemistry to machine learning and ultrafast dynamics", J. Phys. Chem. A, 125, 4276-4293 (2021).

${ }^{5}$ N. A. Besley, "Density functional theory based methods for the calculation of x-ray spectroscopy", Acc. Chem. Res., 53, 1306-1315 (2020).

${ }^{6}$ N. A. Besley, "Modeling of the spectroscopy of core electrons with density functional theory", WIREs Comput. Mol. Sci., pages e1527 (2021).

7 E. Epifanovsky, A. T. B. Gilbert, X. Feng, J. Lee, Y. Mao, N. Mardirossian, P. Pokhilko, A. F. White, M. P. Coons, A. L. Dempwolff, Z. Gan, D. Hait, P. R. Horn, L. D. Jacobson, I. Kaliman, J. Kussmann, A. W. Lange, K. U. Lao, D. S. Levine, J. Liu, S. C. McKenzie, A. F. Morrison, K. D. Nanda, F. Plasser, D. R. Rehn, M. L. Vidal, Z.-Q. You, Y. Zhu, B. Alam, B. J. Albrecht, A. Aldossary, E. Alguire, J. H. Andersen, V. Athavale, D. Barton, K. Begam, A. Behn, N. Bellonzi, Y. A. Bernard, E. J. Berquist, H. G. A. Burton, A. Carreras, K. Carter-Fenk, R. Chakraborty, A. D. Chien, K. D. Closser, V. Cofer-Shabica, S. Dasgupta, M. de Wergifosse, J. Deng, M. Diedenhofen, H. Do, S. Ehlert, P.-T. Fang, S. Fatehi, Q. Feng, J. Gayvert, Q. Ge, G. Gidofalvi, M. Goldey, J. Gomes, C. E. González-Espinoza, S. Gulania, A. O. Gunina, M. W. D. Hanson-Heine, P. H. P. Harbach, A. Hauser, M. F. Herbst, M. Hernandez Vera, M. Hodecker, Z. C. Holden, S. Houck, X. Huang, K. Hui, B. C. Huynh, M. Ivanov, Á. Jász, H. Ji, H. Jiang, B. Kaduk, S. Kähler, K. Khistyaev, J. Kim, G. Kis, P. Klunzinger, Z. Koczor-Benda, J. H. Koh, D. Kosenkov, L. Koulias, T. Kowalczyk, C. M. Krauter, K. Kue, A. Kunitsa, T. Kus, I. Ladjánszki, A. Landau, K. V. Lawler, D. Lefrancois, S. Lehtola, R. R. Li, Y.-P. Li, J. Liang, M. Liebenthal, H.-H. Lin, Y.-S. Lin, F. Liu, K.-Y. Liu, M. Loipersberger, A. Luenser, A. Manjanath, P. Manohar, E. Mansoor, S. F. Manzer, S.-P. Mao, A. V. Marenich, T. Markovich, S. Mason, S. A. Maurer, P. F. McLaughlin, M. F. S. J. Menger, J.-M. Mewes, S. A. Mewes, P. Morgante, J. W. Mullinax, T. Nguyen-Beck, K. J. Oosterbaan, G. Paran, A. C. Paul, S. K. Paul, F. Pavošević, Z. Pei, S. Prager, E. I. Proynov, Á. Rák, E. RamosCordoba, B. Rana, A. E. Rask, A. Rettig, R. M. Richard, F. Rob, E. Rossomme, T. Scheele, M. Scheurer, M. Schneider, N. Sergueev, S. M. Sharada, W. Skomorowski, D. W. Small, C. J. Stein, Y.-C. Su, E. J. Sundstrom, Z. Tao,
J. Thirman, G. J. Tornai, T. Tsuchimochi, N. M. Tubman, S. P. Veccham, O. Vydrov, J. Wenzel, J. Witte, A. Yamada, K. Yao, S. Yeganeh, S. R. Yost, A. Zech, I. Y. Zhang, X. Zhang, Y. Zhang, D. Zuev, A. Aspuru-Guzik, A. T. Bell, N. A. Besley, K. B. Bravaya, B. R. Brooks, D. Casanova, J.-D. Chai, S. Coriani, C. J. Cramer, G. Cserey, A. E. DePrince III, R. A. DiStasio Jr., A. Dreuw, B. D. Dunietz, T. R. Furlani, W. A. Goddard III, S. Hammes-Schiffer, T. Head-Gordon, W. J. Hehre, C.-P. Hsu, T.-C. Jagau, Y. Jung, A. Klamt, J. Kong, D. S. Lambrecht, W. Liang, N. J. Mayhall, C. W. McCurdy, J. B. Neaton, C. Ochsenfeld, J. A. Parkhill, R. Peverati, V. A. Rassolov, Y. Shao, L. V. Slipchenko, T. Stauch, R. P. Steele, J. E. Subotnik, A. J. W. Thom, A. Tkatchenko, D. G. Truhlar, T. Van Voorhis, T. A. Wesolowski, K. B. Whaley, H. L. Woodcock III, P. M. Zimmerman, S. Faraji, P. M. W. Gill, M. Head-Gordon, J. M. Herbert, and A. I. Krylov, "Software for the frontiers of quantum chemistry: An overview of developments in the Q-CHEM 5 package", J. Chem. Phys. (2021).

8 T. Popmintchev, M.-C. Chen, D. Popmintchev, P. Arpin, S. Brown, S. Ališauskas, G. Andriukaitis, T. Balčiunas, O. D. Mücke, A. Pugzlys, A. Baltuška, B. Shim, S. E. Schrauth, A. Gaeta, C. Hernández-García, L. Plaja, A. Becker, A. Jaron-Becker, M. M. Murnane, and H. C. Kapteyn, "Bright coherent ultrahigh harmonics in the keV x-ray regime from mid-infrared femtosecond lasers", Science, 336, 1287-1291 (2012).

9 L. Miaja-Avila, G. C. O’Neil, J. Uhlig, C. L. Cromer, M. L. Dowell, R. Jimenez, A. S. Hoover, K. L. Silverman, and J. N. Ullom, "Laser plasma X-ray source for ultrafast timeresolved x-ray absorption spectroscopy", Struct. Dynam., 2, 024301 (2015).

10 R. Schoenlein, T. Elsaesser, K. Holidack, Z. Huang, H. Kapteyn, M. Murnane, and M. Woerner, "Recent advances in ultrafast x-ray sources", Phil. Trans. R. Soc. A, 377, 20180384 (2019).

11 R. Geneaux, H. J. B. Marroux, A. Guggenmos, D. M. Neumark, and S. R. Leone, "Transient absorption spectroscopy using high harmonic generation: A review of ultrafast $\mathrm{x}$ ray dynamics in molecules and solids", Phil. Trans. $R$. Soc. A, 377, 20170463 (2019).

12 A. Depresseux, E. Oliva, J. Gautier, F. Tissandier, J. Nejdl, M. Kozlova, G. Maynard, J. P. Goddet, A. Tafzi, A. Lifschitz, H. T. Kim, J. Jacquemot, V. Malka, K. T. Phuoc, C. Thaury, P. Rousseau, G. Iaquaniello, T. Lefrou, A. Flacco, B. Vodungbo, G. Lambert, A. Rousse, P. Zeitoun, and S. Sebban, "Table-top femtosecond x-ray laser by collisional ionization gating", Nat. Photonics, 9, 817-822 (2015).

13 J. W. Smith and R. J. Saykally, "Soft x-ray absorption spectroscopy of liquids and solutions", Chem. Rev., 117, 13909-13934 (2017).

14 C. Kleine, M. Ekimova, G. Goldsztejn, S. Raabe, C. Strüber, J. Ludwig, S. Yarlagadda, S. Eisebitt, M. J. J. 
Vrakking, T. Elsaesser, E. T. J. Nibbering, and A. Rouzée, "Soft x-ray absorption spectroscopy of aqueous solutions using a table-top femtosecond soft x-ray source", J. Phys. Chem. Lett., 10, 52-58 (2019).

15 T. Suzuki, "Ultrafast photoelectron spectroscopy of aqueous solutions", J. Chem. Phys., 151, 090901 (2019).

16 A. D. Smith, T. Balčiūnas, Y.-P. Chang, C. Schmidt, K. Zinchenko, F. B. Nunes, E. Rossi, V. Svoboda, Z. Yin, J.-P. Wolf, and H. J. Wörner, "Femtosecond soft-x-ray absorption spectroscopy of liquids with a water-window highharmonic source", J. Phys. Chem. Lett., 11, 1981-1988 (2020).

17 A. Cirri, J. Husek, S. Biswas, and L. R. Baker, "Achieving surface sensitivity in ultrafast XUV spectroscopy: $\mathrm{M}_{2,3^{-}}$ edge reflection-absorption of transition metal oxides", J. Phys. Chem. C, 121, 15861-15869 (2017).

18 E. K. U. Gross and W. Kohn, "Time-dependent density functional theory", Adv. Quantum Chem., 21, 255-291 (1990).

19 E. K. U. Gross, C. A. Ullrich, and U. J. Gossmann, "Density functional theory of time-dependent systems", in Density Functional Theory, E. K. U. Gross and R. M. Dreizler, Eds.; Plenum Press: New York, 1995; pages 149-171.

20 E. K. U. Gross and N. T. Maitra, "Introduction to TDDFT", in Fundamentals of Time-Dependent Density Functional Theory, M. A. L. Marques, N. T. Maitra, F. M. S. Nogueira, E. K. U. Gross, and A. Rubio, Eds., Vol. 837 of Lecture Notes in Physics; Springer-Verlag, 2012; chapter 1 , pages 53-97.

${ }^{21}$ C. A. Ullrich and Z. H. Yang, "A brief compendium of time-dependent density functional theory", Brazilian Journal of Physics, 44(1), 154-188 (2014).

22 R. Baer and L. Kronik, "Time-dependent generalized Kohn-Sham theory", Eur. Phys. J. B, 91, 170 (2018).

23 M. R. Provorse and C. M. Isborn, "Electron dynamics with real-time time-dependent density functional theory", Int. J. Quantum Chem., 116, 739-749 (2016).

${ }^{24}$ X. Li, N. Govind, C. Isborn, A. E. DePrince III, and K. Lopata, "Real-time time-dependent electronic structure theory", Chem. Rev., 120, 9951-9993 (2020).

25 M. E. Casida, "Time-dependent density functional response theory for molecules", in Recent Advances in Density Functional Methods, Part I, D. P. Chong, Ed., Vol. I of Recent Advances in Computational Chemistry; World Scientific: River Edge, NJ, 1995; chapter 5, pages 155-192.

${ }^{26}$ M. E. Casida, "Time-dependent density functional response theory of molecular systems: Theory, computational methods, and functionals", in Recent Developments and Applications of Modern Density Functional Theory, J. M. Seminario, Ed., Vol. 4 of Theoretical and Computational Chemistry; Elsevier, 1996; pages 391-439.

27 F. Furche, "On the density matrix based approach to timedependent density functional response theory", J. Chem. Phys., 114, 5982-5992 (2001).

28 A. Dreuw and M. Head-Gordon, "Single-reference ab initio methods for the calculation of excited states of large molecules", Chem. Rev., 105, 4009-4037 (2005).

${ }^{29}$ F. Furche and D. Rappoport, "Density functional methods for excited states: Equilibrium structure and electronic spectra", in Computational Photochemistry, M. Olivucci, Ed., Vol. 16 of Theoretical and Computational Chemistry; Elsevier: Amsterdam, 2005; chapter 3, pages 93-128.

30 L. S. Cederbaum, W. Domcke, and J. Schirmer, "Manybody theory of core holes", Phys. Rev. A, 22, 206-222
(1980)

31 A. Barth and L. S. Cederbaum, "Many-body theory of core-valence excitations", Phys. Rev. A, 23, 1038-1061 (1981).

32 M. L. Vidal, X. Feng, E. Epifanovsky, A. I. Krylov, and S. Coriani, "New and efficient equation-of-motion coupledcluster framework for core-excited and core-ionized states", J. Chem. Theory Comput., 15, 3117-3133 (2019).

33 M. Stener, G. Fronzoni, and M. de Simone, "Time dependent density functional theory of core electrons excitations", Chem. Phys. Lett., 373, 115-123 (2003).

${ }^{34}$ M. F. Herbst and T. Fransson, "Quantifying the error of the core-valence separation approximation", J. Chem. Phys., 153, 054114 (2020).

35 A. F. Morrison, Z.-Q. You, and J. M. Herbert, "Ab initio implementation of the Frenkel-Davydov exciton model: A naturally parallelizable approach to computing collective excitations in crystals and aggregates", J. Chem. Theory Comput., 10, 5366-5376 (2014).

36 A. F. Morrison and J. M. Herbert, "Low-scaling quantum chemistry approach to excited-state properties via an $a b$ initio exciton model: Application to excitation energy transfer in a self-assembled nanotube", J. Phys. Chem. Lett., 6, 4390-4396 (2015).

37 M. Gao, S. Paul, C. D. Schwieters, Z.-Q. You, H. Shao, J. M. Herbert, J. R. Parquette, and C. P. Jaroniec, "An structural model for a self-assembled nanotube provides insight into its exciton dynamics", J. Phys. Chem. C, 119, 13948-13956 (2015).

38 S. Tussupbayev, N. Govind, K. Lopata, and C. J. Cramer, "Comparison of real-time and linear-response time-dependent density functional theories for molecular chromophores ranging from sparse to high densities of states", J. Chem. Theory Comput., 11, 1102-1109 (2015).

39 Y. Zhu and J. M. Herbert, "Self-consistent predictor/ corrector algorithms for stable and efficient integration of the time-dependent Kohn-Sham equation", J. Chem. Phys., 148, 044117 (2018).

40 K. Lopata and N. Govind, "Modeling fast electron dynamics with real-time time-dependent density functional theory: Application to small molecules and chromophores", J. Chem. Theory Comput., 7, 1344-1355 (2011).

41 K. Lopata, B. E. Van Kuiken, M. Khalil, and N. Govind, "Linear-response and real-time time-dependent density functional theory studies of core-level near-edge x-ray absorption", J. Chem. Theory Comput., 8, 3284-3292 (2012).

42 K. Lopata and N. Govind, "Near and above ionization electronic excitations with non-Hermitian real-time timedependent density functional theory", J. Chem. Theory Comput., 9, 4939-4946 (2013).

43 R. G. Fernando, M. C. Balhoff, and K. Lopata, "X-ray absorption in insulators with non-Hermitian real-time timedependent density functional theory", J. Chem. Theory Comput., 11, 646-654 (2015).

44 J. J. Goings and X. Li, "An atomic orbital based realtime time-dependent density functional theory for computing electronic circular dichroism band spectra", J. Chem. Phys., 144, 234102 (2016).

45 E. S. S. Hansen, S. Kim, J. J. Miller, M. Geferath, G. Morrell, and C. Laustsen, "Fase Padé transform accelerated CSI for hyperpolarized MRS", Tomography, 2, 117-124 (2016).

46 D. Belkić and K. Belkić, "Review of recent applications of the conventional and derivative fast Padé transform for 
magnetic resonance spectroscopy", J. Math. Chem., 57, 385-464 (2019).

47 A. Bruner, D. LaMaster, and K. Lopata, "Accelerated broadband spectra using transition dipole decomposition and Padé approximants", J. Chem. Theory Comput., 12, 3741-3750 (2016).

48 J. M. Herbert, "The quantum chemistry of loosely-bound electrons", in Reviews in Computational Chemistry, A. L. Parill and K. Lipkowitz, Eds., Vol. 28; Wiley-VCH: Hoboken, NJ, 2015; chapter 8, pages 391-517.

49 T.-C. Jagau, K. B. Bravaya, and A. I. Krylov, "Extending the quantum chemistry of bound states to electronic resonances", Annu. Rev. Phys. Chem., 68, 525-553 (2017).

50 M. Thodika, M. Fennimore, T. N. V. Karsili, and S. Matsika, "Comparative study of methodologies for calculating metastable states of small to medium-sized molecules", J. Chem. Phys., 151, 244104 (2019).

51 P. Krause and H. B. Schlegel, "Strong field ionization rates of linear polyenes simulated with time-dependent configuration interaction and an absorbing potential", J. Chem. Phys., 141, 174104 (2014).

${ }^{52}$ P. Krause, J. A. Sonk, and H. B. Schlegel, "Strong field ionization rates simulated with time-dependent configuration interaction and an absorbing potential", J. Chem. Phys., 140, 174113 (2014).

${ }^{53}$ P. Krause and H. B. Schlegel, "Angle-dependent ionization of small molecules by time-dependent configuration interaction and an absorbing potential", J. Phys. Chem. Lett., 6, 2140-2136 (2015).

${ }^{54} \mathrm{P}$. Hoerner and H. B. Schlegel, "Angular dependence of strong field ionization of $\mathrm{CH}_{3} \mathrm{X}(\mathrm{X}=\mathrm{F}, \mathrm{Cl}, \mathrm{Br}$, or I) using time-dependent configuration interaction with an absorbing potential", J. Phys. Chem. A, 121, 5940-5946 (2017).

55 X. Li, S. M. Smith, A. N. Markevitch, D. A. Romanov, R. J. Levis, and H. B. Schlegel, "A time-dependent Hartree-Fock approach for studying the electronic optical response of molecules in intense fields", Phys. Chem. Chem. Phys., 7, 233-239 (2005).

56 S. Dasgupta and J. M. Herbert, "Standard grids for highprecision integration of modern density functionals: SG-2 and SG-3", J. Comput. Chem., 38, 869-882 (2017).

57 N. A. Besley, "Fast time-dependent density functional theory calculations of the x-ray absorption spectroscopy of large systems", J. Chem. Theory Comput., 12, 5018-5025 (2016).

58 P. J. Lestrange, P. D. Nguyen, and X. Li, "Calibration of energy-specific TDDFT for modeling K-edge XAS spectra of light elements", J. Chem. Theory Comput., 11, 29942999 (2015).

59 M. W. D. Hanson-Heine, M. W. George, and N. A. Besley, "Assessment of time-dependent density functional theory calculations with the restricted space approximation for excited state calculations of large systems", Mol. Phys., 116, 1452-1459 (2018).

${ }^{60}$ T. S. Nguyen and J. Parkhill, "Nonadiabatic dynamics for electrons at second-order: Real-time TDDFT and OSCF2", J. Chem. Theory Comput., 11, 2918-2924 (2015).

61 T. S. Nguyen, J. H. Koh, S. Lefelhocz, and J. Parkhill, "Black-box, real-time simulations of transient absorption spectroscopy", J. Phys. Chem. Lett., 7, 1590-1595 (2016).

62 N. A. Besley, M. J. G. Peach, and D. J. Tozer, "Timedependent density functional theory calculations of nearedge x-ray absorption fine structure with short-range corrected functionals", Phys. Chem. Chem. Phys., 11, 1035010358 (2009).

63 N. A. Besley and F. A. Asmuruf, "Time-dependent density functional theory calculations of the spectroscopy of core electrons", Phys. Chem. Chem. Phys., 12, 12024-12039 (2010).

64 M. A. Rohrdanz and J. M. Herbert, "Simultaneous benchmarking of ground- and excited-state properties with long-range-corrected density functional theory", J. Chem. Phys., 129, 034107 (2008).

${ }^{65}$ R. M. Richard and J. M. Herbert, "Time-dependent density-functional description of the ${ }^{1} \mathrm{~L}_{a}$ state in polycyclic aromatic hydrocarbons: Charge-transfer character in disguise?", J. Chem. Theory Comput., 7, 1296-1306 (2011).

66 N. A. Besley, A. T. B. Gilbert, and P. M. W. Gill, "Self-consistent-field calculations of core excited states", J. Chem. Phys., 130, 124308 (2009).

67 R. Sarangi, M. L. Vidal, S. Coriani, and A. I. Krylov, "On the basis set selection for calculations of core-level states: Different strategies to balance cost and accuracy", Mol. Phys., 118, e1769872 (2020).

68 A. E. A. Fouda and N. A. Besley, "Assessment of basis sets for density functional theory-based calculations of core-electron spectroscopies", Theor. Chem. Acc., 137, 6 (2018).

69 M. A. Ambroise and F. Jensen, "Probing basis set requirements for calculating core ionization and core excitation spectroscopy by the $\Delta$ self-consistent-field approach", J. Chem. Theory Comput., 15, 325-337 (2019).

70 M. A. Ambroise, A. Dreuw, and F. Jensen, "Probing basis set requirements for calculating core ionization and core excitation spectra using correlated wave function methods", J. Chem. Theory Comput., 17, 2832-2842 (2021).

71 D. E. Woon and T. H. Dunning Jr., "Gaussian basis sets for use in correlated molecular calculations. V. Core-valence basis sets for boron through neon", J. Chem. Phys., 103, 4572-4585 (1995).

72 Y.-K. Choe, H. A. Witek, J. P. Finley, and K. Hirao, "Identifying and removing intruder states in multireference Møller-Plesset perturbation theory", J. Chem. Phys., 114, 3913-3918 (2001)

73 H. A. Witek, Y.-K. Choe, J. P. Finley, and K. Hirao, "Intruder state avoidance multireference Møller-Plesset perturbation theory", J. Comput. Chem., 23, 957-965 (2002).

74 R. Eisenberg and H. B. Gray, "Noninnocence in metal complexes: A dithiolene dawn", Inorg. Chem., 50, 9741-9751 (2011).

75 R. Sarangi, S. D. George, D. J. Rudd, R. K. Szilagyi, X. Ribas, C. Rovira, M. Almeida, K. O. Hodgson, B. Hedman, and E. I. Solomon, "Sulfur K-edge x-ray absorption spectroscopy as a probe of ligand-metal bond covalency: Metal vs ligand oxidation in copper and nickel dithiolene complexes", J. Am. Chem. Soc., 129, 2316-2326 (2007).

76 Ohio Supercomputer Center http://osc.edu/ark:/19495/f5s1ph73. 